DIVISION OF THE HUMANITIES AND SOCIAL SCIENCES

CALIFORNIA INSTITUTE OF TECHNOLOGY

PASADENA, CALIFORNIA 91125

POLITICAL PARTIES AND ELECTORAL LANDSCAPES

Ken Kollman

University of Michigan

John H. Miller

Carnegie Mellon University

Scott E. Page

California Institute of Technology

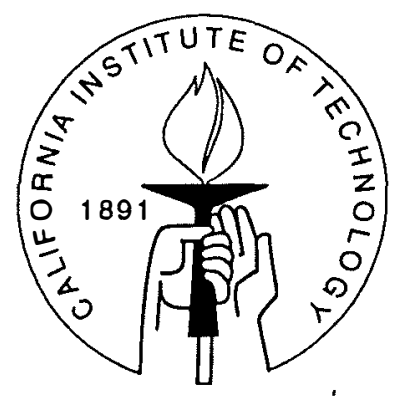

SOCIAL SCIENCE WORKING PAPER 871

November 1993 


\title{
POLITICAL PARTIES AND ELECTORAL LANDSCAPES
}

\author{
Ken Kollman John H. Miller Scott E. Page
}

\begin{abstract}
We study the relationship between voters' preferences and the emergence of party platforms in two-party democratic elections with adaptive parties. In the model, preferences of voters and the opposition party's platform determine an electoral landscape on which the challenging party must adaptively search for votes. We show that changes in the underlying distribution of voters' preferences result in different electoral landscapes which can be characterized by a measure of ruggedness. We find that locally adapting parties converge to moderate platforms regardless of the landscape's ruggedness. Greater ruggedness, however, tempers a party's ability to find such platforms. Thus, we are able to establish a link between the distribution of voters' preferences and the responsiveness of adaptive parties.
\end{abstract}




\title{
POLITICAL PARTIES AND ELECTORAL LANDSCAPES
}

\author{
Ken Kollman John H. Miller Scott E. Page
}

\section{Introduction}

An enduring concern of democratic theory is the extent to which electoral processes encourage political parties to respond to voters' preferences. Scholars have posed various hypotheses about the relationship between voters' preferences and the behavior of parties. For some, sociological and public opinion research suggests that distinct voter ideologies and dispersed policy preferences cause party polarization (Duverger 1963; Sabato 1988; Phillips, 1990). Others rely on spatial election theories to explain party behavior. Spatial theories assume that office-seeking parties try to take policy positions that are as "close" to voters' preferences as possible. Under such an assumption, the distribution of voters' preferences would appear to be central. Downs (1957, p. 140), for example, referring to his single dimensional spatial model, claims "the distribution of voters is a crucial determinant molding a nation's political life ... [and] major changes in it are among the most important political events possible." He asserts that unimodal preference distributions lead parties to adopt similar or identical platforms, while bimodal distributions cause parties to adopt dramatically different platforms (p.118). More recent spatial models have modified Downs' conclusions. If complex preferences become compressed down to a single ideological dimension, regardless of modality, then parties converge toward moderate, or even identical platforms (Enelow and Hinich 1984)

Unfortunately, though spatial models have provided a useful framework for explaining several empirical observations, they have difficulty accounting for policy divergence, challenger parties that lose elections, and the observed stability of platforms in two-party systems. Consider the position-taking behavior of parties in the United States: they alter their messages to appeal to different sets of voters (B. Page 1978); they maintain policy distances from each other on some issues (such as abortion and gun control), and converge to similar positions on other issues (such as foreign policy and social security); and they appear to modify their platforms when voters' preferences for policies change (Nie, Verba, and Petrocik, 1976; Sundquist, 1968.) Spatial voting models have not succeeded entirely in explaining such phenomena. 
With more than one issue dimension, two-party systems have single-point equilibria only if voters are distributed symmetrically - a severely restrictive condition (Plott, 1967). If voters are distributed asymmetrically, equilibrium sets, such as the top-cycle, uncovered, or minmax sets, can be large, or even encompass the whole space (McKelvey, 1976; Kramer, 1977; Ordeshook, 1986; Schofield, Grofman, and Feld, 1988). Only McKelvey (1986) has addressed the connection between preference distributions and the outcomes of electoral competition. He formalizes a bound on the size of the uncovered set as a function of the amount of asymmetry of voter preferences. His result states that less symmetry leads to a larger uncovered set. Therefore, a party with the capability of locating in the uncovered set has greater freedom to select platforms, creating the interpretation that less symmetric voter ideal points allow rational parties to select less moderate platforms.

In this paper, we shall demonstrate with a formal model how, under plausible assumptions, the behavior of political parties changes when voters' preference distributions are altered. Following Kollman, Miller, and Page (1992), we use a model of two-party competition where parties are adaptive organizations competing for votes in a multidimensional issue space. Parties respond to popularity polls by incrementally adapting their platforms. Our model explores the ability of parties, facing a variety of voters' preference distributions, to find winning platforms. We consider classes of voter preference distributions that vary in the level of ideological consistency and the strengths attached to moderate and extreme positions.

In the model, preferences of voters and the opposition party's platform determine what we call an electoral landscape on which the challenging party adaptively searches for votes. We show that changes in the underlying distribution of voters' preferences result in different electoral landscapes that can be characterized by measures of ruggedness and slope. We find that locally adapting parties converge to moderate platforms regardless of the landscape's characteristics. We also find that greater ruggedness, which is correlated with less slope, restricts a party's ability to find such platforms. This leads to greater platform separation. Thus, we are able to establish a link between distributions of voters' preferences and the responsiveness of adaptive parties.

\section{Adaptive Parties}

Standard spatial voting models, which rely on the assumption of fully-informed rational political actors, imply some disturbing conclusions. McKelvey (1976) shows that under quite general conditions, majority-supported policy proposals include the entire space of policy positions. ${ }^{1}$ Therefore, a challenging party can always find a winning platform in a multi-dimensional issue space, and we would not expect systems of competing, fullyinformed rational parties to ever converge to stable platforms. Note also that a further

\footnotetext{
${ }^{1}$ Specifically, McKelvey shows that the top cycle set encompasses the entire space of platforms. This result pertains to the aggregation of preferences, and not the performance of a particular democratic institution.
} 
implication of the fully-informed optimizing perspective is that a challenging party can always defeat an incumbent.

In reality, parties may not act "as if" they are fully-informed and capable of selecting an optimal platform. To account for party limitations, we consider parties that locally adapt from their current platform. There are several explanations which support this assumption. First, a party may have limited information and foresight about voter preferences, so their search for new platforms may be severely restricted. Second, a party may be tethered to policy positions for ideological reasons, including the constraints of party activists and contributors (Aldrich and McGinniss, 1989). Third, voters may be wary of a party that moves across the ideological spectrum quickly in search of votes. Finally, organizations tend to adhere closely to established norms and codes of behavior (Dahl and Lindblom, 1953), and thus are unlikely to engage in large platform changes.

The picture that emerges is one of parties gathering information and choosing the best among those platforms that are "close" to their existing platforms. In this respect our model is similar in spirit to Schofield (1978), who demonstrated with a formal model that there always exists a dense trajectory among winning platforms. That is, one can always find a sequence of close platforms that will lead a political party from one winning platform to the next. Thus, one might think that even with incrementally-changing parties the unsatisfying conclusions inherent in the fully-informed rational choice models still hold. In Schofield's model parties incrementally advance the incumbent's platform as opposed to their own historically determined platform, which is the case in our model. It follows that if parties begin with platforms that are not spatially close to one another, then our model and Schofield's model may lead to very different dynamic trajectories.

To understand adaptive party behavior, we follow the approach suggested by Holland and Miller (1991) and analyze the behavior of parties using artificial adaptive agents (AAA). Using AAA models, we can investigate the relationship between optimization and adaptation. AAA models allow us to create an "artificial world" in which we can explore systems of flexible, well-defined agents, and quickly generate, refine and test new hypotheses. In these models, one attempts to discover the generic patterns of system behavior that emerge due to the adaptation. ${ }^{2}$

Once one accepts the notion that parties are limited in their ability to find appealing platforms, new research questions emerge. The local environment facing each party- the link between small modification in a party's platform and the number of votes the party receives-now becomes central. Recall that the preferences of voters and the opposition party's platform determine the number of votes a party receives. As parties adapt new, nearby platforms in an attempt to capture votes, they alter the environment facing the other party. In this paper, we investigate the dynamic interplay of platforms. What routes do the parties follow? Do they "move" towards similar places in platform space? Do they ever get stuck on local optima? How quickly do they move? How normatively

\footnotetext{
${ }^{2}$ In the complex systems literature, these resulting patterns of system level behavior are often referred to as emergent phenomena.
} 
appealing are the resulting outcomes - are the platforms. selected by adaptive parties better than arbitrarily chosen platforms?

\section{The Model}

Following standard spatial models, our voters have perfect information about parties' platforms. Each voter attaches an integer valued strength and ideal position to each issue. Strength measures the issue's relative importance to the voter. A voter considers an issue irrelevant if the voter's strength is equal to zero on that issue. The ideal position denotes the voter's preferred position.

Thus, an integer valued vector of length $2 n$ (where $n$ equals the number of issues) fully characterizes a voter's preferences. There are $k \in\{0,1, \ldots, k-1\}$ positions on each issue and $s \in\{0,1, \ldots, s-1\}$ strengths. The utility to a voter from a party's platform, $y \in\{0,1, \ldots, k-1\}^{n}$, equals the negative of the squared weighted Euclidean distance, with weights determined by strengths. Let $s_{j i}$ denote the strength and $x_{j i}$ the ideal point of the $j$ th voter on the $i$ th issue. A voter's utility from platform $y$ is then given by

$$
u_{j}(y)=-\sum_{i=1}^{n} s_{j i}\left(x_{j i}-y_{i}\right)^{2} .
$$

A voter casts a ballot for the party whose platform generates the higher utility, and the party obtaining the most votes wins the election.

We begin an electoral sequence with the creation of two randomly assigned initial party platforms. These are referred to as party ideal points. One party is arbitrarily chosen to be the incumbent, and its platform remains fixed during the first election. During the campaign prior to each election, the challenger party tests platform variations using polls of randomly selected voters. ${ }^{3}$ After this polling, the challenger party chooses the platform that maximizes its expected vote total. The parties then compete for election and the winning party becomes the fixed incumbent for the next election. The losing party becomes the challenger and undertakes polls in order to determine a winning platform. Often parties fail to locate winning platforms after the finite campaign. If so, the incumbent remains, and the challenger adapts another new platform. This sequence of adaptations and elections continues through several elections.

\subsection{Adaptive Search Techniques}

Our parties adapt to polling information using three benchmarks of adaptive behavior: a genetic algorithm, hill-climbing, and random search. ${ }^{4}$ A genetic algorithm is a population

\footnotetext{
${ }^{3}$ In Kollman, Miller, and Page (1992), parties were able to poll all voters.

${ }^{4}$ Our use of three different search algorithms ensures more robust results. We have chosen algorithms which satisfy two criteria. First, they have been widely used in discrete nonlinear optimization, which
} 
based adaptive search algorithm that mimics evolutionary learning (Holland 1975). A genetic algorithm begins with a randomly generated population of platforms similar to the party's existing platform. These platforms are then selectively reproduced biased toward those that fair better against the incumbent party's platform. A random subset of the reproduced platforms then undergo an exchange of sequences of positions (crossover) and arbitrary changes on some positions (mutation). The platforms are then tested against the incumbent's platform and a new cycle of reproduction and modification is begun. Each application of the algorithm is referred to as a generation, and the number of generations corresponds to two units of campaign length. Hill-climbing operates on a single platform rather than a population of platforms. At each step, the algorithm randomly generates and tests a new platform in the neighborhood of the current platform. If the new platform is better than the current one, it becomes the new current platform. This process continues with each generation and test of a platform counting as one unit of campaign length. Random search generates multiple platforms in the neighborhood of the existing platform and chooses the best one. Under random search, every new platform generated is considered one unit of campaign length. For each algorithm, we experimented with campaign lengths of between ten and one hundred.

The three adaptive techniques offer plausible analogs to party search behavior. The genetic algorithm approximates the process of a political party undergoing internal debate over policy positions. Candidates borrow ideas from each other and win or lose primaries based on how they match up against the incumbent. Occasionally an activist introduces a new idea. Hill-climbing is intended to represent a party that fine-tunes the policy positions of its candidate using polling results and focus groups. Random search represents a party that chooses the best candidate from among volunteers. The random search algorithm is not a flattering metaphor of party decision making, but we believe that it offers a possible lower bound on organizational intelligence.

\subsection{Preference Distributions}

We consider two major classes of preference distributions: those where voters maintain varying levels of consistency across ideological positions, and those with systematic variations in issue strength. We alter voters' preferences by correlating voters' ideal positions on different issues and by correlating a given issue's strength and position. Correlating voter's ideal positions on different issues alters what we call an ideology. Voters can have uniform ideologies, where their ideal positions are uniformly distributed across all issues, or they can have consistent ideologies, where ideal positions are correlated across issues. To create consistent ideologies, we randomly assign an ideological base to each voter and require that all ideal positions on other issues lie within one position of the base. For example, if the ideological base for a voter is 3 , then all ideal positions lie in the set

provides us with priors on their performance. Second, as we suggest in the next section, each algorithm represents a plausible description of how parties might select platforms. 


\section{$\{2,3,4\}^{5}$}

We also considered three types of correlations between strengths and ideal positions: independent, centrist, or extremist preferences. With independent preferences, a voter's strengths on issues are independent of the voter's ideal positions on issues. Centrist preferences occur when a voter attaches greater strengths to issues with moderate ideal positions, and extremist preferences occur when voters attach greater strengths to those issues with extreme ideal positions. To illustrate, consider the case of nine positions per issue $\{0,1, \ldots, \delta\}$ and three strengths $\{0,1,2\}$. Centrist voters assign high strength $(s=2)$ to issues with ideal positions $\{3,4,5\}$, low strength $(s=1)$ to issues with ideal positions in $\{1,2,6,7\}$, and no strength $(s=0)$ to issues with ideal positions $\{0,8\}$. In extremist preference distributions, voters attach greatest strength to issues on which they have extreme ideal positions. Issues with ideal positions in $\{0,1,7,8\}$, receive high strength $(s=2)$,issues in $\{2,3,5,6\}$ receive low strength $(s=1)$, and an issue with ideal position $\{4\}$ is considered by the voter to be irrelevant $(s=0)$. Since there are two types of ideologies and three types of strength-ideal position correlations, we consider six possible distributions of voter preferences:

Table 1 here

As should be evident, these preference profiles are neither complete nor empirically accurate pictures of political attitudes. Rather, they suggest a set of biases voters might have when they decide how to vote. Our extremist voters place substantial weight on certain subsets of issues on which they take extreme views. One may think of abortion or gun control activists in the United States as extremist voters: the competing parties positions on these voters' pet issue has a huge impact on whom these voters support. In contrast, our centrist voters place little weight on issues on which they have extreme views. For.example, on issues such as the reciting of the pledge of allegiance, funding for the arts, or the personal life of a party's candidate, voters may prefer positions outside of the mainstream and yet not attach much weight to these issues.

\subsection{Measures of Party Performance}

In section 5, we analyze the formation of platforms when adaptive parties face voters with various preference distributions. Ultimately, we want to know how parties, in their efforts

\footnotetext{
${ }^{5}$ If a voter's ideal base position lied on the boundary, in this case in the set 0,8 , then all other ideal positions were within one position of the ideal base position but only in one direction. We also experimented with another version of ideological voters. For these voters, we randomly selected an initial issue (issue 1) and its ideal position, and then requiring the next issue (issue 2) to have an ideal position within plus or minus one the previous issue's ideal position. In turn, a voter's ideal position on issue $3 \mathrm{had}$ to be within one of her issue 2 ideal position and so on. The results for this type of ideological preferences proved indistinguishable from those generated by the consistent ideology described in'the text.
} 
to attract votes, move across the platform space. To facilitate this analysis, we measure party movement and platform responsiveness in a variety of ways. Over the series of elections, we trace the trajectory of winning party platforms based on several measures. In the definitions, we refer to the platform $y_{\text {median }}$, which consists of the median position on each issue. For example, if 3 is the median position on issue 2 then $y_{\text {median,2 }}$ is set equal to 3 .

Separation equals the Euclidean distance between the current platforms of the two parties.

Distance from Median equals the Euclidean distance between the winning party's platform and $y_{\text {median. }}$.

Centrality of a platform $y$ equals

$$
\frac{\sum_{j=1}^{V} u_{j}\left(y_{\text {median }}\right)}{\sum_{j=1}^{V} u_{j}(y)}
$$

where $V$ equals the number of voters.

This normalization sets centrality $\left(y_{\text {median }}\right)=1$. Though we attach no normative significance to the median itself as an outcome, higher centrality means that the platform is closer to the weighted center of voters' preferences. Centralities close to one represent strong utilitarian outcomes. Note, however, that centralities across different distributions of voters' preferences cannot be compared directly because each preference distribution generates a distinct centrality distribution.

\section{The Electoral Landscape}

Adaptive parties attempt to improve their vote totals by searching locally over the space of platforms. Recall that the percentage of the vote a platform receives depends on the incumbent's platform and the preferences of the voters. To explain adaptive parties' responses, we introduce the notion of an electoral landscape. Like geographic landscapes, electoral landscapes have points of high and low elevation. By assigning each platform an elevation equal to the percentage of the vote won by the platform, we can interpret party behavior as a. search for (electoral, not necessarily moral!) high ground.

Notions of electoral landscapes are implicit in models with fully-informed, rational parties, but the landscape's underlying structure are usually ignored. ${ }^{6}$ In these models,

\footnotetext{
${ }^{6}$ The aforementioned work of Mckelvey (1986) and Schofield (1978) are notable exceptions.
} 
agents are able to perform vast surveys of the landscape and to move immediately to the point of highest elevation. In our model, agents are able to only look locally and must move slowly. Thus, the explicit details of the landscape become an important feature of our model.

Adaptive parties' knowledge of the electoral landscape is constrained in two ways. First, they poll only a sample of the electorate and not all voters. Therefore, a platform's elevation, as perceived by the party, may differ from its actual elevation. Of particular importance is when the error caused by sampling results in the incorrect ranking of two platforms, as when an actual decrease in votes is perceived as an increase, or vice versa. Second, adaptive parties do not have estimates of all platforms. In the simulations we discuss, the number of polls never exceeds one hundred, which, in each case, represents substantially less than $1 \%$ of all platforms.

Figures 1 and 2 provide intuition on the impact of the electoral landscape on platform formation. These figures represent projections of two issue dimensions (from a multidimensional problem) on to a voting landscape for centrist and extremist preferences respectively. The horizontal axes represent different platform positions on issues one and two (with all other issue positions fixed). The altitude gives the percentage of the vote received by the party if it advocates the corresponding pair of issue positions. In our model, a party finds itself located somewhere on the horizontal plane, and is allowed to perform local searches for better platforms. Hill-climbing parties, for example, take a sequence of random "steps" and move when the new location is higher than the old. If the landscape is smooth with a single peak, then such steps would lead parties to a similar platform regardless of where the party started. If, however, the landscape is rugged, such steps could cause parties to be trapped on isolated local peaks, unable to cross adjacent valleys and ascend to superior platforms. It follows that we may see adaptive parties with substantial platform separation.

Figure 1 about here

Figure 2 about here

We conclude this section with a description of the formation of electoral landscapes, which provides intuition as to how variations in the distribution of voter preferences alters the slope and the ruggedness of electoral landscapes. We begin with the case of a single voter and a projection of policy preferences onto a two-dimensional issue space. In Figure 3, $v$ is the ideal point for the single voter and $y_{I}$ denotes the incumbent's fixed platform. Using the voter's utility function we can draw an indifference ellipse (I-llipse), such that for all points on the I-llipse the voter achieves utility equal to that given by $y_{I}$. Given the utility function the voter achieves greater utility from platforms that are closer to $v$, thus the challenger will win the vote if and only if its platform is contained within the I-llipse. An I-llipse, $I_{0}$, is shown in Figure 3 for the case when the two platforms offer 
the voter identical utility on the issues not projected on the diagram. If the incumbent's platform offers a different utility level on the unprojected issues, then the I-llipse can be easily adjusted to reflect this differential (it will lie interior of $I_{0}$ if the unprojected issues favor the incumbent and exterior of $I_{0}$ if they favor the challenger).

Figure 3 about here

Now, to form the electoral landscape, draw the appropriate I-llipse for each voter. The "elevation" of each platform is given by the percent of all possible I-llipses that contain the platform. Alternatively, one could view the creation of the landscape as a process by which the interior of each I-llipse is coated with a thick layer of paint (signifying a vote for the challenger). Areas of great elevation, i.e. with many layers of paint, correspond to regions of the issue space which attract many votes.

Different preference patterns create distinct I-llipses and, perforce, fundamentally different electoral landscapes. For example, high strength for a particular issue implies I-llipses that are elongated along the other issue dimension. That is, with high strength voters prefer to stay close to their ideal position on that issue, while compromising on other less important issues. Under extremist preferences, the high strength issues are those with ideal points near the edge of the diagram, and thus one would expect to find elongated I-llipses (running parallel to the nearest edge) in these areas (see Figure 4a). Under centrist preferences, the I-llipses will elongate for those ideal positions near the middle of the axis. These I-llipses will elongate parallel to the other issue axis (see Figure $4 \mathrm{~b})$.

Figures $4 a, 46$ about here

Extremist and centrist preferences imply very different landscapes. With extremist preferences, the I-llipses formations result in numerous areas of high elevation that are sparsely distributed across the landscape (near the corners). These landscapes will therefore have substantial ruggedness. Centrist preferences create landscapes that have a lot of overlap occurring across the middle of the two axes. This causes a landscape to be formed with a, single, central peak. Independent preferences have less regularity than the other two types we consider, as strengths and ideal points are uncorrelated. Thus, the shape of the I-llipses are unconstrained by the voter's ideal point. This results in a landscape formed by combining voters of all different types of characteristics, and thus one can think of a convolution of the various landscapes types discussed above, along with other forms of asymmetric I-llipses not discussed above.

Finally, we want to consider landscapes formed by ideologically consistent voters. Our analysis is simplified by the fact these voters have ideal positions that are near one another on each issue: their icleal points lie along or near the $n$-dimensional diagonal. 
Since our centrist and extremist preferences both tie the strength to the ideal point, such voters will have ideal points with similar strengths, and therefore will have circular Illipses. The radius of such I-llipses will be large towards the extremes and small towards the center for centrist voters, and the opposite for extremists. Further, the compression of the voter ideal points in a small region of the space causes greater regularity. For independent preferences this will smooth the landscape appreciably. In sum, ideological consistent voters should dampen the ruggedness across all types of landscapes.

\section{Results}

In an effort to ensure robust findings, we ran simulations of the model for all six combinations of preference distributions over a range of parameter values. In the findings presented below, we analyze the model when there are ten issues $(n=10)$, nine positions per issue $(k=9)$, three strengths per issue $(s=3)$, and campaigns of length $40 .^{7}$ Strengths and positions are correlated as described in section 3. The results presented are from an environment with 2501 voters and in which parties' polls sample a random selection of 251 voters. ${ }^{8}$ All of the results reported here appear robust to reasonable changes in the parameter values.

To foreshadow the results, we find that adaptive parties evolve moderate platforms that are spatially separate from one another across all types of preference distributions. We also find that platform evolution, in particular the type of platform and speed of convergence, does depend on the underlying preference distribution. Our analysis unfolds in two stages. We first show that variations in preferences result in fundamentally different electoral landscapes. Then we find a link between landscape characteristics and adaptive party behavior.

We characterize electoral landscapes according to two characteristics, ruggedness and slope. Ruggedness equals the percentage of positions in the interior which are onedimensional local maxima or minima. A platform has a one-dimensional local maximum (minimum) on an issue $\mathrm{i}$ at interior position $k$ if when holding the rest of the platform unchanged, no increase in votes occurs when altering issue i's position to either $k+1$ or $k-1$. Intuitively, more ruggedness should lead to slower adaptation, as parties are more likely to get trapped in local optima. ${ }^{9}$ We consider two measures of slope, both of which rely on the notion of a 1-neighbor platform. The set of 1-neighbors of a platform $y$ consists of those platforms which differ from $y$ by exactly one position on exactly one issue.

\footnotetext{
${ }^{7}$ These parameters were chosen to be consistent with existing empirical research, although they are within ranges where we observed no qualitative changes in outcomes. For example, we experimented with campaign lengths between 10 and 100. We also considered ideological parties: those that contingent on winning the election try to stay close to their initial "ideal" platform. The results for ideological parties generally agree with those reported here.

${ }^{8}$ These numbers create a sampling error of approximately $5 \%$.

${ }^{9}$ Ruggedness is a perhaps the simplest measure of the complexity of a landscape. For a discussion of the complexity of discrete function spaces and alternative complexity measures see Page (1993).
} 
The average slope of a landscape equals the average difference (in absolute value) in vote total between a platform and its 1-neighbors. The gradient equals the average maximum vote difference between a platform and its 1-neighbors. We found ruggedness and both measures of slope to be inversely related. Given this inverse relationship; hereafter, we focus our discussion on the ruggedness of landscapes. ${ }^{10}$

Figure 5 gives the average ruggedness across the different types of preference distributions. The preferences can be listed in order from those that form the most to the least rugged landscapes as follows: Independent-Uniform, Independent-Consistent, Extremist-Uniform, Extremist-Consistent, Centrist-Uniform, and Centrist-Consistent. ${ }^{11}$ The intuition outlined in the previous section appears valid: centrist preferences form landscapes that are the least rugged while independent preferences form the most rugged landscapes. Consistency of preferences reduces ruggedness across all landscape types. Note that as the parties evolve their platforms, ruggedness declines slightly, implying that extreme parties move toward moderate platforms slower than parties already near the "center" of voters' preferences. With these measures of landscape characteristics we can now analyze party behavior.

Figure 5 about here

A central finding is that adaptive parties tend to move toward moderate platforms regardless of the distribution of voters' preferences and the search algorithm employed. Figure 6 illustrates this result for hill-climbing parties. ${ }^{12}$ For all types of preferences, adaptive parties, even when limited to sample polls of voters, quickly move to regions of high social utility. In every case, by the fourth election winning parties' platforms were located within the top $1 \%$ of all platforms as measured by centrality. This tendency to adapt platforms of high social utility does not imply platform convergence in the formal sense. The resulting platforms, although close to the median, often advocate positions that are away from the median on particular issues.

Figure 6 about here

Party platforms also maintain a degree of spatial distance from one another (see Figure 7) and do not appear to lock into "equilibrium" positions. Though party platforms tend toward moderate regions, they continually wander these neighborhoods. Moreover, the rate at which parties adapt toward the central region, as well as the region's spatial radius

\footnotetext{
${ }^{10}$ The inverse relationship can be verified by measuring the correlation coefficients between the two measures of slope and ruggedness. For example, with hillclimbing parties after the fourth election the correlation coefficient between average slope and ruggedness was -0.245 for landscapes formed by voters with Independent-Uniform preferences. Similar results hold for other preference distributions.

${ }^{11}$ Only the differences between centrist and other distributions are statistically significant at $95 \%$.

${ }^{12}$ Similar results hold for parties who adapt using genetic algorithms and random search.
} 
vary according to the electoral landscape. This second feature echoes McKelvey's (1976) aforementioned theoretic result and can be observed in Figure 7 with the flattening of the lines at different levels of separation.

We also find that adaptive parties cannot always locate winning platforms. The probability of winning decreases with the number of elections, from approximately $100 \%$ of the time in the first election down to approximately $25 \%$ (on average) by the tenth election. While optimization models require explicit assumptions about incumbency advantages, here such an advantage emerges as a natural outcome of the adaptive search process. As the platforms evolve, the landscape facing each party changes. As shown above, our examples indicate that there is some decrease in ruggedness, which would imply that it is easier for adaptive parties to travel around the landscape without getting trapped on local peaks. However, our examples also show that the slope is decreasing over time, implying greater difficulty in increasing vote totals for a given platform alteration.

\section{Figure 7 about here.}

Figures 6 and 7 also indicate that landscape characteristics effect adaptive party behavior. Linking the behavior observed in these figures, with the underlying landscape characteristics, we find that in general, landscapes with lower ruggedness allow faster adaptation to more moderate platforms that are closer to one other. Thus, centrist preferences will cause competing parties to adapt very moderate platforms that are near one another, while independent or extremist preferences keep party platforms spatially separated with less moderation. The more rugged the landscape, the more likely parties are to get caught on local optima. Thus, on these landscapes initial positions will be very important and path dependent behavior will emerge.

Several other characteristics of the platforms deserve attention. One question regarding consistent voters was whether they compel parties to adapt more consistent platforms. If so, parties would converge to platforms with symmetric positions across all of the issues. We find little support for this hypothesis in our numerical simulations: adaptive parties do not become significantly more consistent when adapting on landscapes formed by consistent voters. ${ }^{13}$

The three search algorithms differ in their abilities to adapt platforms on rugged landscapes. Genetic algorithms have been promoted by many researchers primarily be-

${ }^{13}$ To test this we introduced the following measure of consistency:

$$
c(x)=1-\frac{\operatorname{var}(x)}{M a x v a r}
$$

where $\operatorname{var}(x)$ is the variance of the positions in the platform $x$, and Maxvar equals the highest possible platform variance Consistency always lies on the unit interval and varies inversely with the variance in the platform's positions. We found no significant differences in the consistency of platforms for parties confronting consistent and uniform ideologies. 
cause they perform well on complex, nonlinear problems (Holland 1975). Similar to other population based search techniques, genetic algorithms identify and attempt to exploit patterns present in good solutions, in this case good platforms. It comes as no surprise that on more rugged landscapes the genetic algorithm performs comparatively better than the other two techniques Hill-climbing parties often get caught at a local optima, never visiting more attractive regions of platform space. Finally, though random search adapts most slowly on a simple single peaked landscape, it often outperforms hill-climbing on more rugged landscapes because it samples a larger number of initial points before taking its step, so it is more likely to take high payoff paths initially. ${ }^{14}$ Our examples show that parties using genetic algorithms adapt more quickly away from their initial platform than do those using hill-climbing or random search. Nevertheless, by the tenth election, all types of parties have adapted approximately the same distance away from their initial platforms.

\section{Discussion}

To summarize our findings, the ability of adaptive parties to locate winning platforms depends on the ruggedness and slope of electoral landscapes. We find that extremist and independent preferences lead to slower party convergence than centrist preferences, and that consistent ideologies lead to slower party convergence, especially when preferences are centrist. Moreover, locally adapting and spatially separated parties can often become temporarily stuck at local peaks, and thus be unable to defeat an incumbent. These findings appear to hold for a wide range of parameter values.

The increased ruggedness of landscapes formed by extremist preferences and consistent ideologies may explain some reluctance by contemporary American parties to budge from platform positions. When voters attach greatest strength to those issues on which they take extreme views, or when ideological consistent voters loom large in campaigns, parties appear to converge slowly to moderate positions. On issues where voters attach more significance to centrist positions, parties appear to adapt quickly to similar, moderate positions. By relating different preference distributions to party behavior, our model can lead to more fundamental theories of elections. Moreover, relationships between preferences and outcomes should lend themselves to empirical testing.

While extremist preferences and consistent ideologies tend to create more rugged electoral landscapes, in general, convergence results in spatial voting models with adaptive

\footnotetext{
${ }^{14}$ Hill- climbing and random search are similar in that they both randomly sample from their current best known position and move to better positions. The difference is that random search first samples a large number of neighboring points and then takes the best one, while hill-climbing samples sequentially and moves as soon as a better point emerges. Thus, for a given number of samples, hill-climbing will be able to move further uphill if there is a smooth single-peaked landscape. However, if the landscape is rugged, hill-climbing can take off in the wrong direction and get stuck, while random search will move slower, but each step has a much higher probability of having a greater increase in value. Note that when there are few uphill directions, both algorithms behave in a similar manner.
} 
parties seem relatively robust to changes in voters' preference distributions. Even extremist preferences and consistent ideologies lead to parties taking fairly moderate platforms within a few elections. Our findings tend to support Down's analytical intuition that in two-party democratic elections, rational parties tend to move toward moderate platforms. To infer moderate voter preferences from an electoral system with two moderate parties may be a mistake. Political moderation and slight party separation in two-party systems might be as much attributable to the structural incentives imposed by the democratic process as to the moderation of voters' preferences. Though the notion of adaptive parties competing on an electoral landscape contrasts with the more traditional notion of rational parties optimally locating in an issue space, we view the two approaches as complementary. The robustness of rational actor models can be tested with more flexible AAA modeling techniques. Especially when models become complex, and analytical solutions become difficult or impossible to derive, numerical experiments offer a helpful alternative. We envision these techniques as having the potential to open up new areas for investigation and as a complement to traditional methods.

The authors wish to thank Roger B. Myerson, David Austen-Smith, Tim Feddersen, Jeffrey Banks, and Stan Reiter for helpful comments. Grants from Sun Microsystems and from the Center for Mathematical Studies in Economics and Management Science are gratefully acknowledged. Computer programs are available from the authors upon request. 


\section{$7 \quad$ References}

Aldrich, John and Michael McGinniss. 1989. "A Model of Party Constraints on Optimal Candidate Positions." Mathematical and Computer Modeling. 12:437-450.

Dahl, Robert and Charles Lindblom 1953. Politics, Economics, and Welfare. New York: Harpers.

Downs, Anthony. 1957. An Economic Theory of Democracy. New York: Harper and Row.

Duverger, Maurice. 1963. Political Parties. New York: John Wiley \& Sons.

Enelow, James and Melvin Hinich. 1984. The Spatial Theory of Voting. Cambridge: Cambridge University Press.

Holland, John. 1975. Adaptation in Natural and Artificial Systems. Ann Arbor: University of Michigan Press.

Holland, John, and John H. Miller. 1991. "Artificial Adaptive Agents in Economic Theory." American Economic Review, Papers and Proceedings. 81:365-70.

Kollman, Ken, John H. Miller, and Scott E. Page. 1992. "Adaptive Parties and Spatial Elections," American Political Science Review. 86:929- 37.

Kramer, Gerald. 1977. "A Dynamical Model of Political Equilibrium." Journal of Economic Theory. 15:310-34.

McKelvey, Richard D. 1976. "Intransitivities in Multidimensional Voting Models and Some Implications for Agenda Control." Journal of Economic Theory. 12:472-482.

McKelvey, Richard D. 1986. "Covering, Dominance, and the Institution-Free Properties of Social Choice." American Journal of Political Science. 30:283-314.

Nie, Norman H., Sidney Verba, and John R. Petrocik. 1976. The Changing American Voter. Cambridge, MA: Harvard University Press.

Ordeshook, Peter. 1986. Game Theory and Political Theory. Cambridge: Cambridge University Press.

Page, Benjamin. 1978. Choices and Echoes In Presidential Elections. Chicago: University of Chicago Press.

Page, Scott, E. 1993. "Covers: A Contour Based Measure of Nonlinearity." mimeo. California Institute of Technology.

Plott, Charles. 1967. "A Notion of Equilibrium and Its Possibility Under Majority Rule." American Economic Review. 79:787-806.

Phillips, Kevin. 1990. The Politics of Rich and Poor. New York: Random House. 
Sabato, Larry. 1988. The Party's Just Begun. Glenview, IL: Scott Foresman.

Schofield, Norman. 1978. "Instability of Simple Dynamic Games." Review of Economic Studies. 45:575-594.

Schofield, Norman, Bernard Grofman, and Scott Feld. 198s. "The Core and the Stability of Group Choice in Spatial Voting Games." American Political Science Review. 82:195-211.

Sundquist, James L. 1968. Politics and Policy: The Eisenhower, Kennedy, and Johnson Years. Washington, D.C.: Brookings Institute. 